\title{
Reductions in transdiagnostic factors as the potential mechanisms of change in treatment outcomes in the Unified Protocol: a randomized clinical trial
}

\author{
Sahel Khakpoor, ${ }^{1}$ Jahangir Mohammadi Bytamar, ${ }^{2}$ Omid Saed ${ }^{3}$ \\ ${ }^{1}$ Master of Clinical Psychology, Education and Treatment Center of Beheshti Hospital, Zanjan University of Medical Sciences, Zanjan; \\ ${ }^{2}$ Master of Clinical Psychology, Education and Treatment Center of Valiasr Hospital, Zanjan University of Medical Sciences, Zanjan; \\ ${ }^{3}$ Department of Clinical Psychology, Faculty of Medicine, Zanjan University of Medical Sciences, Zanjan, Iran
}

Correspondence: Omid Saed, Department of Clinical Psychology, Faculty of Medicine, Zanjan University of Medical Sciences, Zanjan, Iran.

Tel. +982433534500 .

Fax +982433534500

E-mail: o.saed@zums.ac.ir

Acknowledgements: the authors would like to thank Social Determinant of Health Research Center of Zanjan University of Medical Sciences. They also thank all participants who contributed to the present study.

Citation: Khakpoor, S., Mohammadi Baytmar, J., \& Saed, O. (2019). Reductions in transdiagnostic factors as the potential mechanisms of change in treatment outcomes in the Unified Protocol: a randomized clinical trial. Research in Psychotherapy: Psychopathology, Process and Outcome, 22(3), 402-412. doi: 10.4081/ ripppo.2019.379

See online Appendix for additional materials.

Contributions: OS and SK carried out and design the experiment; SK wrote the manuscript with support from OS; JMB analyzed the data. All authors discussed the results and commented on the manuscript.

Conflict of interest: the authors declare no potential conflict of interest.

Funding: the work was supported by Social Determinant of Health Research Center of Zanjan University of Medical Sciences.

Clinical trials: the study is registered in Iranian Registry of Clinical Trials (registration number: IRCT2017072335245N1).

Note: this article is part of the master's thesis of the first author of Zanjan University of Medical Sciences (reference number: A-11924-2).

Received for publication: 29 January 2019.

Revision received: 18 May 2019.

Accepted for publication: 21 June 2019.

This work is licensed under a Creative Commons Attribution NonCommercial 4.0 License (CC BY-NC 4.0).

${ }^{\circ}$ Copyright: the Author(s), 2019

Licensee PAGEPress, Italy

Research in Psychotherapy:

Psychopathology, Process and Outcome 2019; 22:402-412

doi:10.4081/ripppo.2019.379

\begin{abstract}
Transdiagnostic approaches emphasize on the share underlying features of emotional disorders. In their view, these transdiagnostic factors play an important role in the etiology, maintenance, and treatment of emotional disorders. This study aimed to investigate the transdiagnostic factors as the potential mechanisms of change in the Unified Protocol (UP) for the transdiagnostic treatment of emotional disorders outcomes. The present study is a randomized clinical trial. Twenty-six individuals were selected based on the Beck anxiety inventory and Beck depression inventory and randomly assigned into two groups of control and treatment $(n=13)$. The treatment group received 20 one-hour individual UP sessions. Beck Depression Inventory, Beck Anxiety Inventory, Difficulty in emotion regulation scale, Intolerance of Uncertainty scale and Acceptance and Action questionnaire were carried out in all three phases. UP enhances the difficulty in emotion regulation (large effect size, SEsg=1.81), intolerance of uncertainty (SEsg=1.91), and experiential avoidance (SEsg=1.78). In addition, the results of linear regression show the association between changes in anxiety and depression with changes in transdiagnostic factors. The difficulty in emotion regulation, intolerance of uncertainty and experiential avoidance can be considered as the potential mechanism of change in improving UP outcomes.
\end{abstract}

Key words: Difficulty in emotion regulation; Intolerance of uncertainty; Experiential avoidance; Unified Protocol; Mediators.

\section{Introduction}

Unified Protocol (UP) is a transdiagnostic treatment that has recently been developed in the treatment of a wide range of emotional disorders (Barlow, Allen, \& Choate, 2016). This protocol was designed in response to the disorder-specific treatments' challenges and aimed to provide a more cost-effective treatment for emotional disorders as well as improve comorbid conditions. Studies supported the effectiveness of this therapeutic protocol in the treatment of a wide range of emotional disorders (Lopez et al., 2015; Maia, Braga, Nunes, Nardi, \& Silva, 2013; Saed, Masjedi, Taremian, Bakhtyari, \& Morsali, 2016). Meta-analysis suggest large effect size (SEsg=1.059) for transdiagnostic treatments at post-treatment and follow-up (Pearl \& Norton, 2016). 
UP uses cognitive-behavior therapies (CBT) underlying principles and integrates them with evidence-based psychological treatment techniques, such as cognitive restructuring of maladaptive appraisals, changing maladaptive emotiondriven behaviors, preventing emotional avoidance, and exposing with emotional interoceptive and situational cues (Norton \& Paulus, 2016). This treatment tends to identify and target common factors beyond the traditional diagnostic criteria as well as factors that can be seen in more than one disorder. These underlying factors, known as transdiagnostic factors, refer to common psychopathological processes among mental disorders (Aldao, 2012), and their form and function are considered to be the same among different disorders. Considering that transdiagnostic factors increase the vulnerability of emotional disorders, it seems that improving these factors plays an important role in the treatment of emotional disorders.

One of the common transdiagnostic factors among emotional disorders is difficulty in using adaptive emotion regulation strategies. The emotion regulation refers to cognitive and behavioral processes that change the frequency, intensity, duration, and emotional reactions (Berking et al., 2008). The results showed that there is a relationship between maladaptive emotion regulation strategies and a wide range of psychological problems, such as symptoms of anxiety disorders, eating disorders and depression (Berking \& Wupperman, 2012; Naragon-Gainey, McMahon, \& Chacko, 2017). Indeed, difficulty in emotion regulation is an important underlying factor in the etiology and maintenance of emotional disorders. Therefore, it seems that improving different emotion regulation skills, and not just reducing a particular emotion, can facilitate the treatment and reduce patients' symptoms. Evidence suggested that therapeutic programs that target emotion regulation can be used to treat a wide range of disorders (Barlow, 2013; Ehrenreich-May \& Bilek, 2012). In this regard, UP is unique due to its emphasis on the way in which the emotions are experienced and responded (Trosper, Buzzella, Bennett, \& Ehrenreich, 2009). This protocol serves as an emotion-focused treatment, using techniques such as present-focused non-judgmental awareness (module 3), cognitive restructuring (module 4), and situational emotion exposures (module 6 and 7), improve the individual's relationship with his/her emotions and replace maladaptive emotion regulation strategies with more adaptive ones (Ehrenreich, Fairholme, Buzzella, Ellard, \& Barlow, 2007).

Intolerance of Uncertainty (IU) is another underlying factor that has been proposed in the etiology and treatment of emotional disorders. IU includes a set of negative beliefs about uncertainty and is defined as the fear of unknowns and the tendency to consider ambiguous events as unacceptable and threatening, irrespective of the probability of their occurrence (Carleton, 2012). In addition to a negative reaction to uncertainty, IU involves the inabil- ity to cope with ambiguity and changes. Although this construct was initially developed to explain worry in generalized anxiety disorder (GAD), results indicated that IU plays an important role in etiology and maintenance of wide range of anxiety and depression symptoms, and can be considered as a transdiagnostic factor (Allan et al., 2018; Paulus, Talkovsky, Heggeness, \& Norton, 2015). In addition, given the transdiagnostic and transtherapy nature of IU (McEvoy \& Erceg-Hurn, 2016), it seems that cognitive behavior therapies can potentially reduce this underlying factor in various emotional disorders. Research has shown that treatments that do not focus explicitly on targeting IU, can improve this construct in individuals with depression, social anxiety disorder (SAD) (Mahoney \& McEvoy, 2012), and GAD (van der Heiden, Muris, \& van der Molen, 2012). Boswell, Thompson-Hollands, Farchione, and Barlow indicated that UP can improve this underlying factor among different diagnostic groups (Boswell, Thompson-Hollands, Farchione, \& Barlow, 2013). UP tries to increase the tolerance of uncertainty through methods such as present-focused, nonjudgmental awareness (module 3), as well as exposure to situations that cause uncertainty (module 6 and 7) and improves emotional disorders symptoms in this group of people.

Another underlying factor that is discussed in the psychopathology of emotional disorders is Experiential Avoidance. Experiential avoidance is characterized by the avoidance of unpleasant internal experiences and consists of two parts: unwillingness to remain in contact with unwanted inner experiences and attempts to avoid events that elicit these experiences (Hayes, Wilson, Gifford, Follette, \& Strosahl, 1996). At first, this construct was the primary goal of Acceptance and Commitment Therapy (ACT); but, further research showed that various treatments could also affect change through alterations in experiential avoidance (Espejo, Gorlick, \& Castriotta, 2016; Gloster et al., 2014). In addition, studies demonstrated that experiential avoidance is more a psychological vulnerability (Feldner, Zvolensky, Eifert, \& Spira, 2003; Karekla, Forsyth, \& Kelly, 2004) rather than the consequence of psychological problems, and it can be considered as an important transdiagnostic factor among various emotional disorders.

Malicki and Ostaszewski proposed experiential avoidance as the basic functional dimension of the psychopathology of anxiety and depression. They stated that the experiential avoidance is, in fact, a collection of the behaviors that the person displays in order to avoid unwanted inner experiences, and thus can be considered as an underlying and transdiagnostic factor in the psychopathology and treatment of mental disorders (Malicki \& Ostaszewski, 2014). Espejo, Gorlick, and Castriotta discussed that transdiagnostic group CBT could significantly reduce experiential avoidance in veterans (Espejo et al., 2016). Given that awareness and exposure techniques directly target avoidance, UP can be considered as 
an appropriate treatment option in reducing experiential avoidance among patients with emotional disorders.

According to evidence and in order to identify UP effect mechanisms, it is essential to examine the role of transdiagnostic factors as the mediator of treatment outcomes. In this regard, the first goal of this study was to examine the efficacy of UP in reducing anxiety, depression over the course of treatment. We hypothesized that UP can reduce anxiety, depression, significantly. The second goal of this study was to examine changes in difficulty in emotion regulation, intolerance of uncertainty, and experiential avoidance in individuals with emotional disorders. Based on prior research, it was hypothesized that UP can reduce difficulty in emotion regulation, intolerance of uncertainty and experiential avoidance. In addition, the present study aimed to determine if changes in these transdiagnostic factors are associated with treatment improvements. Consistent with available transdiagnostic models and existing theories, it was hypothesized that change in difficulty in emotion regulation, intolerance of uncertainty and experiential avoidance over the course of treatment would be associated with changes in emotional symptoms.

\section{Methods}

The present study is double-blind randomized clinical trial and had been approved by the Ethics Committee of Zanjan University of Medical Sciences (reference number: ZUMS.REC.1396.143). It is also registered in Iranian Registry of Clinical Trials (registration number: IRCT2017072335245N1) There are additional registered secondary outcome measures in trial registration document: Negative Affect, Perceived Control, and Occupational and Social Performance. Given that these measures can be considered as antecedents or consequence of developing emotional symptoms, our team decided not to report these variables in this manuscript. All participants enrolled agreed to take part in the trial and informed consent was obtained from them.

\section{Participants}

Participants were selected in two steps among Zanjan University of Medical Sciences students $(\mathrm{N}=3500)$. At first, a sample of 315 individuals was recruited by convenience sampling method, and the Beck Anxiety Inventory (BAI) and Beck Depression Inventory (BDI-II) were conducted. In the second step, eligible individuals were selected based on depression scores (20 to 28) and anxiety scores (16 to 30) (149 people). Eventually, according to the inclusion and exclusion criteria, a sample of 26 individuals was selected. In trial registration document the target sample size was 32 . However, during sampling process only 26 individuals remained for randomization which is likely to make the study to be underpowered
$(<.80$, alpha $=0.05)$. Figure 1 summarizes the sampling process. Inclusion criteria included residence in Zanjan city during the research, willingness to participate in the research and having a principal diagnosis of any anxiety or depression disorders (assessed using the Anxiety Disorders Interview Schedule for DSM-IV-Lifetime Version). Exclusion criteria included having a DSM-IV diagnosis of bipolar disorder, schizophrenia and schizoaffective disorder, history of psychiatric problems, history of psychological interventions (especially more than 5 sessions of CBT), absences of more than two sessions, or not participating in the evaluation process.

These 26 individuals were randomly assigned to treatment $(n=13)$ and control group $(n=13)$ who were selected by the second author of this study based on Random Number Generator 3.1 software (the therapist was blind to randomized condition). During the treatment, two patients from the treatment group and 1 from the control group were excluded from the study.

The treatment group $(\mathrm{n}=11)$ consisted of $27.3 \%$ male and $72.7 \%$ female with an average age of 24.27 years $(\mathrm{SD}=2.65)$. In terms of educational status, $27.3 \%$ of the participants in the treatment group were undergraduate students, $27.3 \%$ were master students and $45.5 \%$ were medical students. The control group $(\mathrm{n}=12)$ included $16.7 \%$ male and $83.3 \%$ female with an average age of 26.67 years $(\mathrm{SD}=23.5), 33.3 \%$ were undergraduate students, $33.3 \%$ were master students and $33.3 \%$ were medical students. There was no significant difference between the two groups in gender $\left(X^{2}=0.379, \mathrm{P}=0.538\right)$ and education $\left(X^{2}=0.354, \mathrm{P}=0.838\right)$ and in the mean age $(\mathrm{t}=-1.36$, $\mathrm{P}=0.187$ ); which indicates the homogeneity of both groups in demographic variables. Participants' principal diagnosis included: obsessive-compulsive disorder (OCD, $\mathrm{n}=3$ ), generalized anxiety disorder $(\mathrm{GAD}, \mathrm{n}=7)$, social anxiety disorder $(\mathrm{SOC}, \mathrm{n}=5)$, panic disorder $(\mathrm{PD}, \mathrm{n}=1)$, major depression disorder (MDD, $\mathrm{n}=5$ ). Two participants had co-principal diagnoses (equal severity): SOC and PD, OCD and GAD. Comorbid disorders included MDD $(n=5)$ and OCD $(n=1)$. None of the participants were using psychiatric drugs during the evaluation and treatment phases.

\section{Measures}

\section{Anxiety Disorders Interview Schedule for DSM-IV-Lifetime Version}

This program is a semi-structured diagnostic interview designed to assess the existence and severity of anxiety, mood, somatoform disorders, as well as mental health history. This scale also includes a short screening for psychotic symptoms and alcohol and substance use. Large-scale evaluations of this tool have been carried out and strong support has been provided for using diagnostic reliability (Brown \& Barlow, 2013). It has very good internal reliability for anxiety and mood disorders (A, B, \& M, 2013). 


\section{Beck Depression Inventory}

This 21-item inventory was designed by Beck, Steer, and Carbin in 1996 to measure the severity of depression over the past two weeks. Studies conducted on psychometric properties of BDI-II in different countries show that this inventory has acceptable reliability. Beck et al. reported a high internal consistency for BDI-II $(\alpha=0.91)$ and retest reliability of 0.93 in a week. In a study done in Iran on non-clinical and clinical samples, internal consistency coefficients were 0.90 and 0.89 , respectively and the test-retest coefficients in the non-clinical sample were 0.94 (Ghassemzadeh, Mojtabai, Karamghadiri, \& Ebrahimkhani, 2005).

\section{Beck Anxiety Inventory}

This 21-item inventory was designed by Beck et al. in 1998 to measure the severity of anxiety in adults and adolescents. Beck et al. obtained Cronbach's alpha coefficient of 0.93 and five-week test-retest reliability coefficient for this inventory 0.83 (de Beurs, Wilson, Chambless, Goldstein, \& Feske, 1997). In Iran, an adequate internal con- sistency of BAI $(\alpha=0.92)$ and test-retest $(r=0.83)$ has been reported (Rafiei \& Seifi, 2013).

\section{Difficulty in Emotion Regulation Scale}

This scale was developed by Gratz and Roemer in 2004 with 36 items and 6 subscales for measuring emotion dysregulation and emotional self-regulation strategies. The subscales of this scale include the lack of acceptance of emotional responses, difficulty in performing purposeful behavior, difficulty controlling impulse, lack of emotional awareness, limited access to emotion regulation strategies, and lack of clarity of emotion. High internal consistency $(\alpha=0.93)$ and adequate two-week testretest reliability $(\mathrm{r}=0.85)$ has been reported for this scale (Gratz \& Roemer, 2004). The reliability of the Persian version of Difficulty in Emotion Regulation Scale (DERS) also varies from 0.79 to 0.91 , using the Cronbach's alpha coefficient, and the reliability of its test-retest is between 0.86 and 0.88 after a week (Khanzadeh, Saeediyan, Hosseinchari, \& Edrissi, 2012).

\section{Assessed for eligibility}
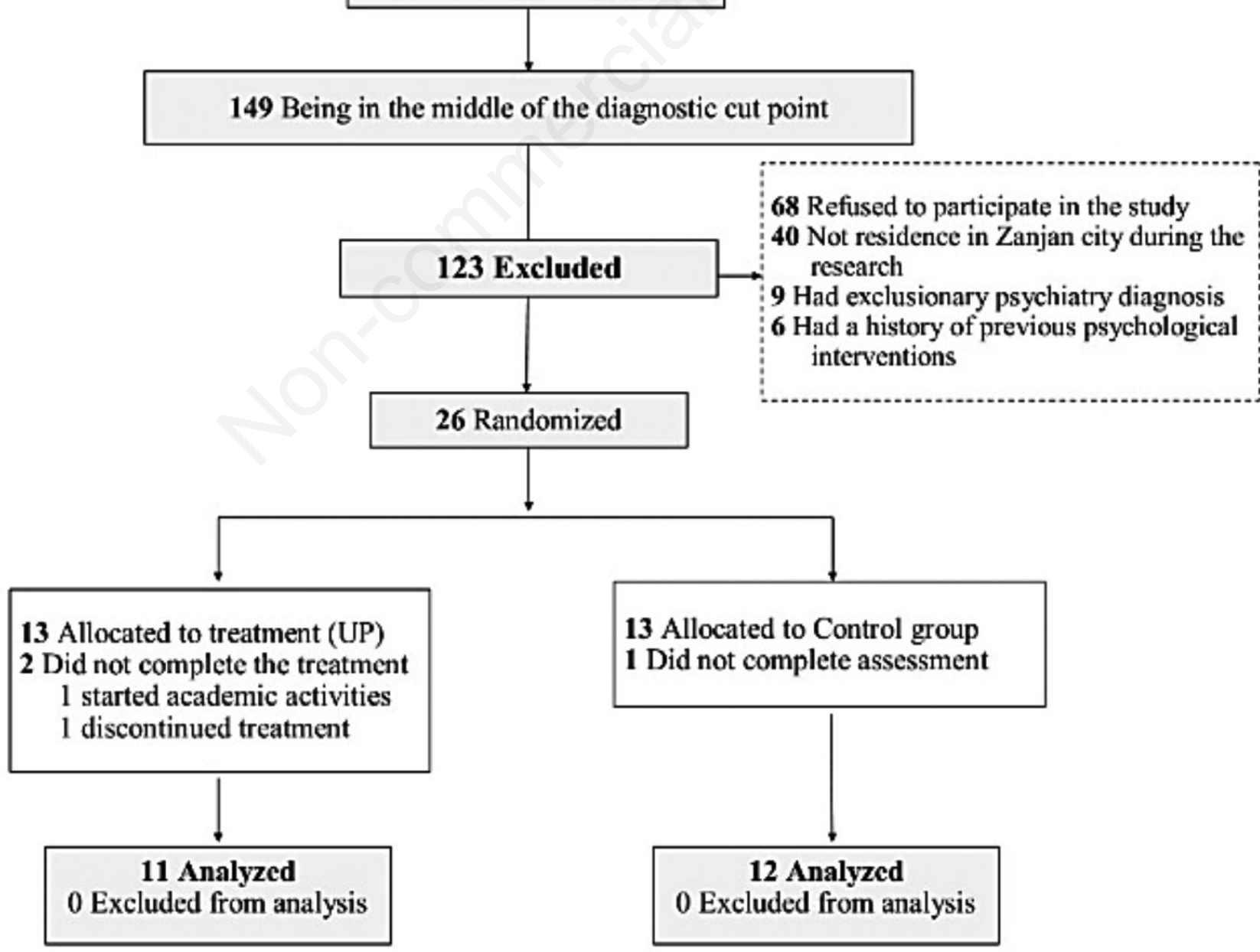

Figure 1. CONSORT diagram illustrating participant flow during the study. 


\section{Acceptance and Action Questionnaire-II}

This questionnaire was developed by Bond et al. in 2011 with 10 items to measure experiential avoidance. Items are rated on a 7-point Likert scale ranging from 1 (never true) to 7 (always true). It has demonstrated adequate internal consistency $(\alpha=0.84)$, and test-retest reliability ( $\mathrm{r}=0.70$, over 12 months) (Bond et al., 2011). Internal consistency for the Persian version was 0.89 and split-half reliability was 0.71 (Abasi, Fti, Molodi, \& Zarabi, 2012).

\section{Intolerance of Uncertainty Scale}

This scale is a 12 -item version of the original 27 -item Intolerance of Uncertainty Scale (IUS-12) designed in 2007 by Carleton et al. to measure the ability to tolerate uncertainty in ambiguous situations, behavioral and cognitive responses to uncertainty, and effort to control future events. Cronbach's alpha ranged from 0.92 to 0.94 . The correlation of the short form with the original version has been obtained from 0.94 to 0.96 (Carleton, Norton, \& Asmundson, 2007).

\section{Procedure}

The treatment group received 20 one-hour individual psychotherapy sessions. UP consists of 8 modules, which include: motivational enhancement (module 1), psychoeducation regarding the function of emotions (module 2), development of present-focused, nonjudgmental awareness (module 3, core), cognitive flexibility (module 4, core), attenuation of emotional and behavioral avoidance (module 5, core), increased tolerance of physical sensations (module 6, core), situational emotion exposures (module 7, core) and, finally, relapse prevention (module 8). The treatment was provided by the master student of clinical psychology (the first author of this article) who received the necessary training for $\mathrm{CBT}$ and transdiagnostic treatment. The therapist was blind to randomization. Moreover, the assessments and analyses were conducted by independent evaluators who were blind to treatment condition allocation.

All treatment sessions were conducted under the supervision of a professor of clinical psychology (the second author of this article). In order to determine the appropriateness of the UP sessions, therapeutic sessions were audiotaped and were randomly examined by the supervisor. In addition, weekly meetings were conducted between the therapist and the professor of clinical psychology to supervise treatment adherence. The supervisor indicated that the therapist adhered to the treatment protocol, and appropriately delivered UP modules. In addition, patient fielded the questions appropriately and elicited relevant examples.

\section{Data analysis}

SPSS version 20 was used to perform statistical analyses. A series of repeated measure ANOVAs were calcu- lated to investigate the efficacy of UP in reducing anxiety, depression, difficulty in emotion regulation, IU and experiential avoidance from pre-test to post-test and twomonth follow-up in the treatment and control groups. Bonferroni post hoc test was used to compare time phases two-by-two in independent variables. Hedges effect size was calculated to determine the magnitude of change from pre- to post-treatment and two-month follow-up in a treatment group more accurately. One-way analysis of variance (ANOVA) was applied to compare treatment and control groups on change scores from pre- to post-treatment and pre-treatment to follow-up. Finally, linear regression analysis was conducted on the treatment group to investigate the role of transdiagnostic factors in reducing anxiety and depression. In order to small sample size, Bootstrapping with 1000 resamples was performed to ensure the robust nature of statistically significant results. Bootstrapping accounts were considered meaningful if the upper and lower limits of the Confidence Interval (95\% CI) did not encompass zero (Hayes, 2009). All the analyses were performed only on the participants who completed the study (23 individuals).

\section{Results}

Table 1 presents pre-treatment, post-treatment, and 2month follow-up scores. The 2 (group: Treatment $v s$ Control) $\times 3$ (time point: pre-treatment $v s$ post-treatment $v s$ 2-month follow-up) repeated measures ANOVAs revealed significant main effects for group (BAI: $F[1,27]=5.42$, $\eta^{2}=0.21, \mathrm{P}=0.03$; BDI: $F[1,27]=13.51, \eta^{2}=0.39, \mathrm{P}=0.001$; DERS: $\quad F[1,27]=15.97, \quad \eta^{2}=0.43, \quad \mathrm{P}<0.001 ; \quad$ IUS: $F[1,27]=14.58, \eta^{2}=0.41, \mathrm{P}<0.001$; Acceptance and Action Questionnaire-II (AAQ): $\quad F[1,27]=13.83, \quad \eta^{2}=0.40$, $\mathrm{P}=0.001$ ), and time (BAI: $F[1,27]=7.03, \eta^{2}=0.25$, $\mathrm{P}=0.005$; BDI: $F[1,27]=7.67, \eta^{2}=0.27, \mathrm{P}=0.001$; DERS: $F[1,27]=9.21, \eta^{2}=0.31, \mathrm{P}<0.001$; IUS: $F[1,27]=10.16$, $\eta^{2}=0.33, \quad \mathrm{P}<0.001 ; \quad$ AAQ: $\quad F[1,27]=6.48, \quad \eta^{2}=0.31$, $\mathrm{P}=0.004)$. Significant interaction effects were also founded in all variables (BAI: $F[1,27]=11.37, \eta^{2}=0.35$, $\mathrm{P}<0.001$; BDI: $F[1,27]=17.67, \eta^{2}=0.46, \mathrm{P}<0.001$; DERS: $F[1,27]=12.73, \eta^{2}=0.38, \mathrm{P}<0.001$; IUS: $F[1,27]=13.97$, $\eta^{2}=0.40, \quad \mathrm{P}<0.001 ; \quad \mathrm{AAQ}: \quad F[1,27]=13.50, \quad \eta^{2}=0.39$, $\mathrm{P}<0.001)$. The result of pairwise comparisons with Bonferroni test indicated a significant reduction in dependent variables' means from pre- to post-treatment and pretreatment to follow-up $(\mathrm{P}<0.05$, Table 2, Figure 1).

In order to examine the interaction effects, one-way ANOVAs conducted on pre- to post-treatment and pretreatment to follow-up. Mean change scores showed that the treatment group achieved a significantly greater magnitude of change than the control group for all measures from pre- to post-treatment (BAI: $F[1,27]=16.14$, $\mathrm{P}<0.001 ; \quad$ BDI: $\quad F[1,27]=20.74, \quad \mathrm{P}<0.001 ; \quad$ DERS: $F[1,27]=18.27, \mathrm{P}<0.001$; IUS: $F[1,27]=17.19, \mathrm{P}<0.001$; AAQ: $F[1,27]=22.60, \mathrm{P}<0.001$ ), and pre-treatment to fol- 
low-up (BAI: $\quad F[1,27]=11.21, \quad \mathrm{P}=0.003 ; \quad$ BDI: $F[1,27]=35.59, \quad \mathrm{P}<0.001 ; \quad$ DERS: $\quad F[1,27]=23.38$, $\mathrm{P}<0.001 ; \quad$ IUS: $\quad F[1,27]=18.80, \quad \mathrm{P}<0.001 ; \quad$ AAQ: $F[1,27]=20.85, \mathrm{P}<0.001)$. Appendix Figure 1 displays mean change scores from pre- to post-treatment for treatment and control groups on all measures.

Table 1 also displays the within-group effect sizes (Standardized Gain, ESsg) for treatment and control groups at post-treatment and 2-month follow- up. Hedges reported effect size of $0.2,0.5$ and 0.8 as small, medium and large, respectively (Ellard, Fairholme, Boisseau, Farchione, \& Barlow, 2010). Pre- to post-treatment withingroup effect sizes for the treatment group were large for all measures (SEsg=1.30-1-91). Post-treatment withingroup effect sizes appeared stable at 2-month follow-up for Treatment group (Figure 2).

In order to investigate the potential mechanism of change in decreasing anxiety and depression, linear regression analysis was conducted on the treatment group. Since the transdiagnostic factors have been showed to overlap with each other (Table 3, Pearson correlation matrix), and because of the multiple linearities between them (Tolerance $<0.3$, VIF $>3.5$ ), we were not allowed to enter all three transdiagnostic factors simultaneously as independent variables in a multiple regression model (Pallant, 2011). Therefore, three regression analyses were conducted to investigate the separate share of each factor in the prediction of depression and anxiety (Table 4).

Results showed that changes in transdiagnostic factors significantly associated with a reduction in anxiety and depression. Change in the difficulty in emotion regulation accounted for $53.3 \%$ of $57.2 \%$ of the variance of the changes in anxiety and depression scores, respectively. Results indicated that change in difficulty in emotion regulation significantly associated with the changes in anxiety $(\beta=0.729$, CI: $0.18-0.48, \mathrm{P}<0.001)$ and depression $(\beta=0.756$, CI: 0.18-0.44, $\mathrm{P}<0.001)$.

Change in IU has also been able to explain $69 \%$ of the variance of anxiety and $42 \%$ of the variance of depression. Results indicated that change in IU significantly associated with the changes in both anxiety $(\beta=0.831, \mathrm{CI}$ : 0.81 $1.56, \mathrm{P}<0.001)$ and depression $(\beta=0.648$, CI: 0.48-1.34, $\mathrm{P}<0.001)$. Finally, change in experiential avoidance accounted for $69.8 \%$ and $57.8 \%$ of the variance of the changes in anxiety and depression scores, respectively. Results showed that change in experiential avoidance significantly associated with changes in anxiety $(\beta=0.835$, CI: $0.51-0.92, P<0.001)$ and depression $(\beta=0.760, \mathrm{CI}$ : 0.34-0.90, $\mathrm{P}<0.001)$.

\section{Discussion}

The first aim of this study was to examine the efficacy of UP in reducing anxiety and depression in individuals with emotional disorders. The initial results showed that the UP could significantly reduce anxiety and depression in the treatment group relative to the control group. This finding is consistent with the underlying theories and therapeutic goals of the UP as well as previous studies (Ellard et al., 2010; Farchione et al., 2012; Pearl \& Norton, 2016) and has provided other empirical evidence to support the efficacy of UP in the treatment of emotional disorders.

The second study aim examined the efficacy of UP in

Table 1. Descriptive statistics and within- and between-group effects on self-report measures for treatment and Control Groups at each assessment.

\begin{tabular}{|c|c|c|c|c|c|c|c|}
\hline \multirow[t]{2}{*}{ Measure and group } & \multirow{2}{*}{$\begin{array}{c}\text { Pre-Treatment } \\
\mathbf{M} \pm \mathbf{S D}\end{array}$} & \multirow{2}{*}{$\begin{array}{c}\text { Post-Treatment } \\
\mathbf{M} \pm \mathrm{SD}\end{array}$} & \multirow{2}{*}{$\begin{array}{c}\text { Follow-up } \\
\mathrm{M} \pm \mathrm{SD}\end{array}$} & \multicolumn{2}{|c|}{ Within-group ESsg } & \multicolumn{2}{|c|}{ Between-group ESsg } \\
\hline & & & & $\begin{array}{c}\text { Pre- to } \\
\text { post-treatment }\end{array}$ & $\begin{array}{l}\text { Pre-treatment } \\
\text { o follow-up }\end{array}$ & Post-treatment & Follow-up \\
\hline \multicolumn{8}{|l|}{ BAI } \\
\hline Treatment $(n=11)$ & $19.82 \pm 11.80$ & $7.91 \pm 5.43$ & $8.09 \pm 3.14$ & 1.30 & 1.36 & -1.46 & -1.56 \\
\hline Control $(n=12)$ & $20.00 \pm 12.79$ & $21.25 \pm 11.77$ & $21.58 \pm 11.83$ & -0.10 & -0.13 & & \\
\hline \multicolumn{8}{|l|}{ BDI } \\
\hline Treatment $(n=11)$ & $15.91 \pm 6.96$ & $5.27 \pm 6.36$ & $5.09 \pm 5.58$ & 1.60 & 1.72 & -1.82 & -2.04 \\
\hline Control $(n=12)$ & $19.67 \pm 10.13$ & $21.92 \pm 11.28$ & $21.83 \pm 10.17$ & -0.21 & -0.21 & & \\
\hline \multicolumn{8}{|l|}{ DERS } \\
\hline Treatment $(n=11)$ & $95.73 \pm 20.14$ & $67.55 \pm 8.74$ & $71.80 \pm 17.52$ & 1.82 & 1.27 & -2.51 & -1.72 \\
\hline Control $(n=12)$ & $107.42 \pm 22.63$ & $109.00 \pm 21.57$ & $110.50 \pm 26.44$ & -0.07 & -0.12 & & \\
\hline \multicolumn{8}{|l|}{ IUS } \\
\hline Treatment $(n=11)$ & $30.18 \pm 5.08$ & $21.27 \pm 4.22$ & $21.09 \pm 5.09$ & 1.91 & 1.79 & -1.87 & -2.02 \\
\hline Control $(n=12)$ & $31.67 \pm 6.36$ & $32.08 \pm 6.99$ & $32.67 \pm 6.32$ & -0.06 & -0.16 & & \\
\hline \multicolumn{8}{|l|}{ AAQ } \\
\hline Treatment $(n=11)$ & $37.00 \pm 9.62$ & $22.55 \pm 6.36$ & $24.64 \pm 8.44$ & 1.77 & 1.37 & -2.71 & -1.52 \\
\hline Control $(n=12)$ & $37.33 \pm 8.08$ & $39.75 \pm 6.33$ & $39.92 \pm 11.47$ & -0.33 & -0.26 & & \\
\hline
\end{tabular}

ESsg, Standardized Gain effect size; BAI, Beck Anxiety Inventory; BDI, Beck Depression Inventory; DERS, Difficulty in Emotion Regulation Scale; IUS, Intolerance of Uncertainty Scale; AAQ, Acceptance and Action Questionnaire. 
reducing difficulty in emotion regulation, IU, and experiential avoidance in patients with emotional disorders. As expected, UP could improve these transdiagnostic factors in individuals with emotional disorders. This finding is consistent with previous studies showed that UP can improve the individuals' responses to the emotional experiences (Gratz, Weiss, \& Tull, 2015; Sauer-Zavala et al., 2012), IU (Boswell et al., 2013; Zemestani, Imani, \& Ottaviani, 2017), and experiential avoidance (Espejo et al., 2016; Thompson-Brenner, Boswell, Espel-Huynh, Brooks, \& Lowe, 2018) in different diagnostic groups.
This finding is also in line with the UP underlying theories and treatment goals. According to the triple vulnerability theory, general vulnerabilities are the common underlying processes in most of the emotional disorders and should be the main focus of treatments. In this regard, UP appears to effectively target these processes it was designed to address and can correct individuals' maladaptive and pathologic strategies using various modules (especially modules 3 to 7 ).

The third aim of this study was to investigate the association between changes in difficulty in emotion regu-

Table 2. The result of pairwise comparisons with Bonferroni test.

\begin{tabular}{|c|c|c|c|c|c|c|c|}
\hline Measure & Time 1 & Time 2 & $\mathbf{M}_{\text {diff }}$ & SE & P-value & Lower CI & Upper CI \\
\hline \multirow[t]{3}{*}{ BAI } & Pre-Treatment & Post-Treatment & 5.33 & 1.64 & 0.004 & 1.92 & 8.74 \\
\hline & & Follow-up & 5.07 & 1.99 & 0.019 & 0.93 & 9.21 \\
\hline & Post-Treatment & Follow-up & -0.26 & 1.04 & 0.806 & -2.41 & 1.89 \\
\hline \multirow[t]{3}{*}{ BDI } & Pre-Treatment & Post-Treatment & 4.19 & 1.41 & 0.007 & 1.25 & 7.13 \\
\hline & & Follow-up & 4.33 & 1.09 & 0.001 & 2.06 & 6.59 \\
\hline & Post-Treatment & Follow-up & 0.13 & 1.24 & 0.916 & -2.45 & 2.72 \\
\hline \multirow[t]{3}{*}{ DERS } & Pre-Treatment & Post-Treatment & 13.30 & 3.48 & 0.001 & 6.06 & 20.54 \\
\hline & & Follow-up & 10.41 & 2.79 & 0.001 & 4.61 & 16.22 \\
\hline & Post-Treatment & Follow-up & -2.87 & 3.46 & 0.413 & -10.08 & 4.30 \\
\hline \multirow[t]{3}{*}{ IUS } & Pre-Treatment & Post-Treatment & 4.25 & 1.12 & 0.001 & 1.91 & 6.58 \\
\hline & & Follow-up & 4.05 & 1.16 & 0.002 & 1.63 & 6.47 \\
\hline & Post-Treatment & Follow-up & -0.20 & 0.88 & 0.821 & -2.02 & 1.62 \\
\hline \multirow[t]{3}{*}{$\mathrm{AAQ}$} & Pre-Treatment & Post-Treatment & 6.02 & 1.77 & 0.003 & 2.33 & 9.71 \\
\hline & & Follow-up & 4.89 & 1.64 & 0.007 & 1.49 & 8.29 \\
\hline & Post-Treatment & Follow-up & -1.13 & 1.91 & 0.561 & -5.10 & 2.84 \\
\hline
\end{tabular}

BAI, Beck Anxiety Inventory; BDI, Beck Depression Inventory; DERS, Difficulty in Emotion Regulation Scale; IUS, Intolerance of Uncertainty Scale; AAQ, Acceptance and Action Questionnaire.

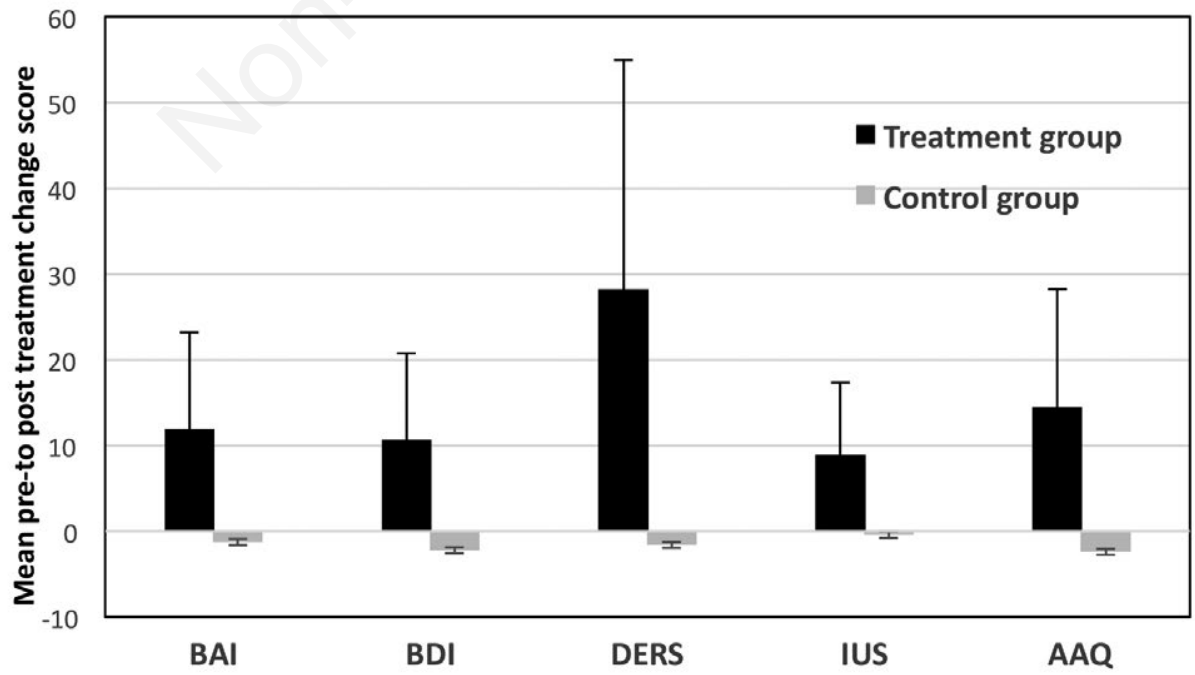

Figure 2. Mean change score and $95 \%$ confidence interval lower and upper bounds for the magnitude of change from pre-treatment to post-treatment on all measures for Treatment and Control Groups. BAI, Beck Anxiety Inventory; BDI, Beck Depression Inventory; DERS, Difficulty in Emotion Regulation Scale; IUS, Intolerance of Uncertainty Scale; AAQ, Acceptance and Action Questionnaire. 
lation, IU, and experiential avoidance and changes in emotional disorders' symptoms. The results showed that there is a connection between improving transdiagnostic factors and reducing anxiety and depression in individuals with emotional disorders after participating in UP.

Improvement in difficulty in emotion regulation strategies has been found to largely associate with changes in anxiety and depression. Review of the research in the field of emotion regulation shows that despite the emphasis of transdiagnostic treatment on the role of emotion regulation, limited studies have been conducted on the mediating role of this factor. Sauer Zavala et al. showed that UP decreased the severity of post-treatment emotional symptoms through the reduction in negative reaction to emotional experiences (Sauer-Zavala et al., 2012). In addition, some studies indicated that other cognitive-behavior therapies can reduce individuals' anxiety and depression symptoms through the improvement in emotion regulation strategies (Berking et al., 2008; Gratz et al., 2015; Suveg, Sood, Comer, \& Kendall, 2009).

People with emotional disorders consider their emotional experiences to be uncontrollable and interpret them as threats. Studies suggest that beliefs associated with control have a direct and significant relationship with the adaptive emotion regulation strategies (Cisler, Olatunji, Feldner, \& Forsyth, 2010). Lopez et al., suggested that adaptive emotion regulation strategies training, improves perceived control in individuals with emotional disorders (López, González, García-Palacios, \& Arbona, 2016). It can be inferred that UP improves perceived control using second-level mediators such as emotion regulation, and thus reduces individuals' anxiety and depression symptoms. It is probable that UP modules 3-5, helps individuals to respond to their emotions in a more adaptive way and moderates their evaluations of their emotions. In addition, modules that expose individuals with the emotions and situations which elicit these emotions (modules 6 and 7), may lead to new situational learning as well as replacing maladaptive emotion regulation strategies with adaptive ones.

On the other hand, results indicate that improving IU is associated with a reduction of anxiety and depression. In line with this finding, various studies suggest that IU can be considered as a potential mediator of treatment outcomes in different cognitive-behavior therapies (Bomyea et al., 2015; McEvoy \& Erceg-Hurn, 2016). However, to the best of our knowledge, limited studies have examined the mediational role of this factor in UP treatment. Boswell et al. showed that changing in IU during treatment with UP could significantly predict the treatment outcomes (Boswell et al., 2013). Studies on group transdiagnostic treatment demonstrated that this treatment can reduce IU, anxiety, and depression in individuals with emotional disorders (Talkovsky \& Norton, 2016, 2018).

When people with emotional disorders face with am-

Table 3. Average, standard deviation and correlation matrix of variables.

\begin{tabular}{lcccccc}
\hline Variables & $\mathbf{M} \pm \mathbf{S D}$ & $\mathbf{2}$ & $\mathbf{3}$ & $\mathbf{4}$ & $\mathbf{5}$ \\
\hline 1. BAI & & $10.19 \pm 5.04$ & $0.710^{* *}$ & $0.729^{* *}$ & $0.831^{* *}$ & $0.835^{* *}$ \\
\hline 2. BDI & $9.33 \pm 3.91$ & 1 & $0.756^{* *}$ & $0.648^{* *}$ & $0.760^{* *}$ \\
\hline Transdiagnostic Factors & 3. DERS & $22.28 \pm 12.65$ & & 1 & $0.783^{* *}$ & $0.850^{* *}$ \\
\hline & 4. IUS & $7.10 \pm 4.4$ & & 1 & $0.734^{* *}$ \\
\hline & 5. AAQ & $11.97 \pm 5.65$ & & & 1 \\
\hline
\end{tabular}

BAI, Beck Anxiety Inventory; BDI, Beck Depression Inventory; DERS, Difficulty in Emotion Regulation Scale; IUS; Intolerance of Uncertainty Scale; AAQ, Acceptance and Action Questionnaire $* * \mathrm{P}<0.001$.

Table 4. Regression analysis for the prediction of change in anxiety and depression.

\begin{tabular}{|c|c|c|c|c|c|c|c|c|c|c|}
\hline \multirow{2}{*}{\multicolumn{2}{|c|}{$\begin{array}{l}\text { Prediction Dependent } \\
\text { variables variables }\end{array}$}} & \multirow[t]{2}{*}{$\mathbf{R}$} & \multirow[t]{2}{*}{ R Square } & \multirow[t]{2}{*}{ F } & \multirow[t]{2}{*}{ P-value of $F$} & \multirow[t]{2}{*}{ Beta } & \multirow[t]{2}{*}{$\mathrm{t}$} & \multirow[t]{2}{*}{ P-value } & \multicolumn{2}{|c|}{$\begin{array}{c}\text { Bootstrap } \\
95 \% \text { CI }\end{array}$} \\
\hline & & & & & & & & & Upper & Lower \\
\hline \multirow[t]{2}{*}{ DERS } & BAI & 0.729 & 0.533 & 23.85 & $<0.001$ & 0.729 & $4.88 * *$ & $<0.001$ & 0.48 & 0.18 \\
\hline & BDI & 0.756 & 0.572 & 28.03 & $<0.001$ & 756 & $5.29 * *$ & $<0.001$ & 0.44 & 0.18 \\
\hline \multirow[t]{2}{*}{ IUS } & BAI & 0.831 & 0.690 & 46.77 & $<0.001$ & 0.831 & $6.84 * *$ & $<0.001$ & 1.56 & 0.81 \\
\hline & BDI & 0.648 & 0.420 & 15.21 & $<0.001$ & 0.648 & $3.89 * *$ & $<0.001$ & 1.34 & 0.48 \\
\hline \multirow[t]{2}{*}{ AAQ } & BAI & 0.835 & 0.698 & 48.47 & $<0.001$ & 0.835 & $6.96^{* *}$ & $<0.001$ & 0.92 & 0.51 \\
\hline & BDI & 0.760 & 0.578 & 28.79 & $<0.001$ & 0.760 & $5.36^{* *}$ & $<0.001$ & 0.90 & 0.34 \\
\hline
\end{tabular}

BAI, Beck Anxiety Inventory; BDI, Beck Depression Inventory; DERS, Difficulty in Emotion Regulation Scale; IUS, Intolerance of Uncertainty Scale; AAQ, Acceptance and Action Questionnaire; $\mathrm{CI}$, Confidence Interval. $* * \mathrm{P}<0.001$. 
biguous conditions, get confused (Dugas, Buhr, \& Ladouceur, 2004) and try to reduce this experience of uncertainty by some strategies like compulsive behaviors, safety behaviors or avoidance. Nevertheless, these attempts to achieve certainty and moderate the situation, contradictory increase uncertainty and distress. In such situation, it seems that UP can modify individuals' perception of uncertainty and ambiguity by using various therapeutic techniques, such as present-focused, nonjudgmental awareness (module 3 ) and cognitive flexibility (module 4). Additionally, it is likely that treatment helps patients to identify and be aware of their maladaptive emotion-driven behaviors (EDBs) and avoidance patterns (module 5). In order to create more adaptive cognitive and behavioral strategies, patients are encouraged to counter patterns of avoidance and EDBs, and expose to the ambiguous situations (module 7).

A remarkable point in the present study was the finding that IU has a stronger association with changes in anxiety than depression. McEvoy and Erceg-Hurn suggested that IU is associated with a larger reduction of repetitive negative thoughts than a number of depressive symptoms (McEvoy \& Erceg-Hurn, 2016). In fact, contrary to the direct relationship between anxiety symptoms and IU, this construct indirectly affects depression symptoms, for example through repetitive negative thoughts (Liao \& Wei, 2011). When individuals with depressive symptoms faced with ambiguous situations, they try to reduce this uncertainty through rumination, which in turn causes and exacerbates depression symptoms. From this perspective, it is possible that in a patient with depression, the existence of a pessimistic certainty is more related to the symptoms than failure to tolerate uncertainty (Miranda \& Mennin, 2007). Another potential explanation for the lack of association between changes in IU and changes in depression symptoms could be due to the strategies used in CBT. During the treatment people with anxiety disorders are often exposed to unknown and obscure situations that are unaware of the consequences. In contrast, people with depression are often encouraged to engage with activities that are already known their benefits and therefore do not face a lot of unknown situations. In addition, the scale used to measure IU in the present study was more sensitive to the strategies used by people with anxiety disorders. Only 4 of the 12 items investigate the strategies of individuals with depression, which can affect the results. Future research is needed to investigate the potential of these explanations more specifically.

Eventually, the results indicated that change in experiential avoidance is associated with a reduction of anxiety and depression. In line with this finding, Espejo et al. showed that group transdiagnostic treatment could reduce negative emotions and fear in veterans by reducing the experiential avoidance (Espejo et al., 2016). Other studies also suggested that different cognitive-behavior therapies may also improve emotional disorders symptoms through alterations in experiential avoidance (Arch et al., 2012; Eustis, Hayes-Skelton, Roemer, \& Orsillo, 2016; Gloster et al., 2014).

People with emotional disorders are trying to avoid unpleasant experiences using maladaptive strategies, such as emotional suppression. In this regard, it is likely that UP help patients to fully experience the present moment (module 3). Emotional and situational avoidance is another maladaptive strategy that helps patients to deal with unpleasant emotional situations. This emotional and situational avoidance causes persistence of emotional disorders symptoms due to avoidance of getting accustomed. Under such circumstances, it is likely that UP identifies and decreases patients' emotional avoidance using various techniques (module 5-7), and so influences levels of emotional disorders symptoms.

This study had several limitations. First, the sample size of the study was small which makes it difficult to generalize the findings. Secondly, the current study only examined anxiety and depression changes in general, and the mechanisms of changes in specific symptoms and comorbid conditions did not demonstrate.

The current study provides evidence of the association between improvement in transdiagnostic factors and reduction of anxiety and depression. Future research should continue to investigate the mediational role of these transdiagnostic factors in larger groups. Furthermore, more research needs to be done to show the exact interaction between these transdiagnostic factors. In addition, identifying mechanisms of the effect of each UP module separately can help to extend and improve this treatment protocol.

\section{Conclusions}

Determining the mechanism of change during participating in UP could be beneficial for clinical decisionmaking and treatment development. It seems that UP can reduce anxiety and depression symptoms as well as transdiagnostic factors. The results propound difficulty in emotion regulation, IU, and experiential avoidance as a potential change process in reducing anxiety and depression in individuals with emotional disorders.

\section{References}

Abasi, E., Fti, L., Molodi, R., \& Zarabi, H. (2013). Psychometric properties of Persian Version of Acceptance and Action Questionnaire -II. Scientific Journal Management System, 3(10), 65-80.

Abdi, R., Bakhshi, A., \& Mahmoud Alilou, M. (2013). Efficacy of Unified Transdiagnostic Treatment on Reduction of Transdiagnostic Factors and Symptoms Severity in Emotional Disorders. Journal of psychological models and methods, 3(13).

Aldao, A. (2012). Emotion regulation strategies as transdiagnos- 
tic processes: a closer look at the invariance of their form and function. Spanish Journal of Clinical Psychology, 17(3), 261-277. doi:10.5944/rppc.vol.17.num.3.2012.11843

Allan, N. P., Cooper, D., Oglesby, M. E., Short, N. A., Saulnier, K. G., \& Schmidt, N. B. (2018). Lower-order anxiety sensitivity and intolerance of uncertainty dimensions operate as specific vulnerabilities for social anxiety and depression within a hierarchical model. Journal of anxiety disorders, 53, 91-99. doi:10.1016/j.janxdis.2017.08.002

Arch, J. J., Eifert, G. H., Davies, C., Vilardaga, J. C. P., Rose, R. D., \& Craske, M. G. (2012). Randomized clinical trial of cognitive behavioral therapy (CBT) versus acceptance and commitment therapy (ACT) for mixed anxiety disorders. Journal of Consulting and Clinical Psychology, 80(5), 750. doi: $10.1037 / \mathrm{a} 0028310$

Barlow, D. H. (2013). Anxiety and Its Disorders, Second Edition: The Nature and Treatment of Anxiety and Panic (D. H. Barlow Ed.): Guilford Publications.

Barlow, D. H., Allen, L. B., \& Choate, M. L. (2016). Toward a Unified Treatment for Emotional Disorders-Republished Article. Behavior Therapy, 47(6), 838-853. doi:10.1016/j.beth. 2016.11.005

Berking, M., \& Wupperman, P. (2012). Emotion regulation and mental health: recent findings, current challenges, and future directions. Curr Opin Psychiatry, 25(2), 128-134. doi:10.1097/YCO.0b013e3283503669

Berking, M., Wupperman, P., Reichardt, A., Pejic, T., Dippel, A., \& Znoj, H. (2008). Emotion-regulation skills as a treatment target in psychotherapy. Behaviour research and therapy, 46(11), 1230-1237. doi:10.1016/j.brat.2008.08.005

Bomyea, J., Ramsawh, H., Ball, T., Taylor, C., Paulus, M., Lang, A., \& Stein, M. (2015). Intolerance of uncertainty as a mediator of reductions in worry in a cognitive behavioral treatment program for generalized anxiety disorder. Journal of anxiety disorders, 33, 90-94. doi:10.1016/j.janxdis.2015.05.004

Bond, F. W., Hayes, S. C., Baer, R. A., Carpenter, K. M., Guenole, N., Orcutt, H. K., Waltz, T., \& Zettle, R. D. (2011). Preliminary psychometric properties of the Acceptance and Action Questionnaire-II: A revised measure of psychological inflexibility and experiential avoidance. Behavior Therapy, 42(4), 676-688. doi:10.1016/j.beth.2011.03.007

Boswell, J. F., Thompson-Hollands, J., Farchione, T. J., \& Barlow, D. H. (2013). Intolerance of Uncertainty: A Common Factor in the Treatment of Emotional Disorders. Journal of clinical psychology, 69(6), 21. doi:10.1002/jclp.21965

Brown, T. A., \& Barlow, D. H. (2013). Anxiety Disorders Interview Schedule for DSM-IV: Life time version and clinical manual (Abolfasl Mohammadi \& B. Birashk, Trans.). Tehran: Neevand Publication.

Carleton, R. N. (2012). The intolerance of uncertainty construct in the context of anxiety disorders: theoretical and practical perspectives. Expert Review of Neurotherapeutics, 12(8), 937-947. doi:10.1586/ern.12.82

Carleton, R. N., Norton, M. P. J., \& Asmundson, G. J. (2007). Fearing the unknown: A short version of the Intolerance of Uncertainty Scale. Journal of anxiety disorders, 21(1), 105117. doi:10.1016/j.janxdis.2006.03.014

Cisler, J. M., Olatunji, B. O., Feldner, M. T., \& Forsyth, J. P. (2010). Emotion regulation and the anxiety disorders: An integrative review. Journal of psychopathology and behavioral assessment, 32(1), 68-82. doi:10.1007/s10862-009-9161-1

de Beurs, E., Wilson, K. A., Chambless, D. L., Goldstein, A. J., \& Feske, U. (1997). Convergent and divergent validity of the Beck Anxiety Inventory for patients with panic disorder and agoraphobia. Depression and anxiety, 6(4), 140-146. doi:10.1002/(SICI) 1520-6394(1997)6:4<140::AID$\mathrm{DA} 2>3.0 . \mathrm{CO} ; 2-\mathrm{G}$

Dugas, M. J., Buhr, K., \& Ladouceur, R. (2004). The Role of Intolerance of Uncertainty in Etiology and Maintenance Generalized anxiety disorder: Advances in research and practice. (pp. 143-163). New York, NY, US: Guilford Press.

Ehrenreich-May, J., \& Bilek, E. L. (2012). The development of a transdiagnostic, cognitive behavioral group intervention for childhood anxiety disorders and co-occurring depression symptoms. Cognitive and Behavioral Practice, 19(1), 4155. doi:10.1016/j.cbpra.2011.02.003

Ehrenreich, J. T., Fairholme, C. P., Buzzella, B. A., Ellard, K. K., \& Barlow, D. H. (2007). The role of emotion in psychological therapy. Clinical Psychology: Science and Practice, 14(4), 422-428. doi:10.1111/j.1468-2850.2007.00102.x

Ellard, K. K., Fairholme, C. P., Boisseau, C. L., Farchione, T. J., \& Barlow, D. H. (2010). Unified Protocol for the Transdiagnostic Treatment of Emotional Disorders: Protocol Development and Initial Outcome Data. Cognitive and Behavioral Practice 17, 88-101. doi:10.1016/j.cbpra.2009.06.002

Espejo, E. P., Gorlick, A., \& Castriotta, N. (2016). Changes in threat-related cognitions and experiential avoidance in group-based transdiagnostic CBT for anxiety disorders. Journal of anxiety disorders, 46, 1-7. doi:10.1016/j.janxdis. 2016.06.006

Eustis, E. H., Hayes-Skelton, S. A., Roemer, L., \& Orsillo, S. M. (2016). Reductions in experiential avoidance as a mediator of change in symptom outcome and quality of life in acceptance-based behavior therapy and applied relaxation for generalized anxiety disorder. Behaviour research and therapy, 87, 188-195. doi:10.1016/j.brat.2016.09.012

Farchione, T. J., Fairholme, C. P., Ellard, K. K., Boisseau, C. L., Thompson-Hollands, J., Carl, J. R., Gallagher, M. W., \& Barlow, D. H. (2012). Unified Protocol for Transdiagnostic Treatment of Emotional Disorders: A Randomized Controlled Trial. Behavior Therapy, 43(3), 666-678. doi:10.1016/j.beth.2012.01.001

Feldner, M. T., Zvolensky, M. J., Eifert, G. H., \& Spira, A. P. (2003). Emotional avoidance: An experimental test of individual differences and response suppression using biological challenge. Behaviour research and therapy, 41(4), 403-411. doi:10.1016/S0005-7967(02)00020-7

Ghassemzadeh, H., Mojtabai, R., Karamghadiri, N., \& Ebrahimkhani, N. (2005). Psychometric properties of a Persian-language version of the Beck Depression InventorySecond edition: BDI-II-PERSIAN. Depress Anxiety, 21(4), 185-192. doi:10.1002/da.20070

Gloster, A. T., Klotsche, J., Gerlach, A. L., Hamm, A., Ströhle, A., Gauggel, S., Kircher, T., Alpers, G. W., Deckert, J., \& Wittchen, H.-U. (2014). Timing matters: Change depends on the stage of treatment in cognitive behavioral therapy for panic disorder with agoraphobia. Journal of Consulting and Clinical Psychology, 82(1), 141. doi:10.1037/a0034555

Gratz, K. L., \& Roemer, L. (2004). Multidimensional assessment of emotion regulation and dysregulation: Development, factor structure, and initial validation of the difficulties in emotion regulation scale. Journal of psychopathology and behavioral assessment, 26(1), 41-54. doi:10.1023/B:JOBA. 0000007455.08539 .94

Gratz, K. L., Weiss, N. H., \& Tull, M. T. (2015). Examining emotion regulation as an outcome, mechanism, or target of 
psychological treatments. Current opinion in psychology, 3, 85-90. doi:10.1016/j.copsyc.2015.02.010

Hayes, A. F. (2009). Beyond Baron and Kenny: Statistical mediation analysis in the new millennium. Communication monographs, 76(4), 408-420. doi:10.1080/03637750903310360

Hayes, S. C., Wilson, K. G., Gifford, E. V., Follette, V. M., \& Strosahl, K. (1996). Experiential avoidance and behavioral disorders: A functional dimensional approach to diagnosis and treatment. Journal of Consulting and Clinical Psychology, 64(6), 1152. doi:10.1037/0022-006X.64.6.1152

Karekla, M., Forsyth, J. P., \& Kelly, M. M. (2004). Emotional avoidance and panicogenic responding to a biological challenge procedure. Behavior Therapy, 35(4), 725-746. doi:10.1016/S0005-7894(04)80017-0

Khanzadeh, M., Saeediyan, M., Hosseinchari, M., \& Edrissi, F. (2012). Factor structure and psychometric properties of difficulties in emotional regulation scale. International Journal of Behavioral Sciences, 6(1), 87-96.

Liao, K. Y. H., \& Wei, M. (2011). Intolerance of uncertainty, depression, and anxiety: The moderating and mediating roles of rumination. Journal of clinical psychology, 67(12), 12201239. doi:10.1002/jclp.20846

López, J. O., González, J. R. B., García-Palacios, A., \& Arbona, C. B. (2016). Influence of vulnerability factors in panic disorder severity. Psicothema, 28(2), 167-173. doi:10.7334/psicothema2015.223

Lopez, M. E., Stoddard, J. A., Noorollah, A., Zerbi, G., Payne, L. A., Hitchcock, C. A., Meier, E. A., Esfahani, A. M., \& Ray, D. B. (2015). Examining the efficacy of the unified protocol for transdiagnostic treatment of emotional disorders in the treatment of individuals with borderline personality disorder. Cognitive and Behavioral Practice, 22(4), 522-533. doi:10.1016/j.cbpra.2014.06.006

Mahoney, A. E. J., \& McEvoy, P. M. (2012). Changes in intolerance of uncertainty during cognitive behavior group therapy for social phobia. Journal of Behavior Therapy and Experimental Psychiatry, 43(2), 849-854. doi:10.1016/j. jbtep.2011.12.004

Maia, A. C. C., Braga, A. A., Nunes, C. A., Nardi, A. E., \& Silva, A. C. (2013). Transdiagnostic treatment using a unified protocol: application for patients with a range of comorbid mood and anxiety disorders. Trends in psychiatry and psychotherapy, 35(2), 134-140. doi:10.1590/S2237-60892013000200007

Malicki, S., \& Ostaszewski, P. (2014). Experiential avoidance as a functional dimension of a transdiagnostic approach to psychopathology. Institute of Psychiatry and Neurology, 23, 61-71. doi:10.1016/j.pin.2014.04.002

McEvoy, P. M., \& Erceg-Hurn, D. M. (2016). The search for universal transdiagnostic and trans-therapy change processes: Evidence for intolerance of uncertainty. Journal of anxiety disorders, 41, 96-107. doi:10.1016/j.janxdis. 2016.02.002

Miranda, R., \& Mennin, D. S. (2007). Depression, generalized anxiety disorder, and certainty in pessimistic predictions about the future. Cognitive therapy and research, 31(1), 7182. doi:10.1007/s10608-006-9063-4

Naragon-Gainey, K., McMahon, T. P., \& Chacko, T. P. (2017). The structure of common emotion regulation strategies: A meta-analytic examination. Psychol Bull, 143(4), 384-427. doi: $10.1037 /$ bul0000093

Norton, P. J., \& Paulus, D. J. (2016). Toward a Unified Treatment for Emotional Disorders: Update on the Science and Practice. Behav Ther, 47(6), 854-868. doi:10.1016/j.beth. 2015.07.002
Pallant, J. (2011). SPSS Survival manual: a step by step guide to data analysis using SPSS. Crows Nest. New South Wales: Allen \& Unwin.

Paulus, D. J., Talkovsky, A. M., Heggeness, L. F., \& Norton, P. J. (2015). Beyond negative affectivity: A hierarchical model of global and transdiagnostic vulnerabilities for emotional disorders. Cognitive Behaviour Therapy, 44(5), 389-405. doi:10.1080/16506073.2015.1017529

Pearl, S. B., \& Norton, P. J. (2017). Transdiagnostic versus diagnosis specific cognitive behavioural therapies for anxiety: A meta-analysis. Journal of anxiety disorders, 46, 11-24. doi:10.1016/j.janxdis.2016.07.004

Rafiei, M., \& Seifi, A. (2013). An investigation into the reliability and validity of beck anxiety inventory among the university students. Iran J Psychiatry Clin Psychol, 7(27), $37-46$.

Saed, O., Masjedi, A., Taremian, F., Bakhtyari, M., \& Morsali, Y. (2016). The efficacy of Transdiagnostic Cognitive Behavior therapy on reducing symptoms severity of Obsessive Compulsive Disorder with co-occurring anxiety and mood disorders. International Journal of Applied Behavioral Sciences, 2(3), 1-10. doi:10.22037/ijabs.v2i4.11194

Sauer-Zavala, S., Boswell, J. F., Gallagher, M. W., Bentley, K. H., Ametaj, A., \& Barlow, D. H. (2012). The role of negative affectivity and negative reactivity to emotions in predicting outcomes in the unified protocol for the transdiagnostic treatment of emotional disorders. Behaviour research and therapy, 50(9), 551-557. doi:10.1016/j.brat.2012.05.005

Suveg, C., Sood, E., Comer, J. S., \& Kendall, P. C. (2009). Changes in emotion regulation following cognitive-behavioral therapy for anxious youth. Journal of Clinical Child \& Adolescent Psychology, 38(3), 390-401. doi:10.1080/153744 10902851721

Talkovsky, A. M., \& Norton, P. J. (2016). Intolerance of uncertainty and transdiagnostic group cognitive behavioral therapy for anxiety. Journal of anxiety disorders, 41, 108-114. doi:10.1016/j.janxdis.2016.05.002

Talkovsky, A. M., \& Norton, P. J. (2018). Negative affect and intolerance of uncertainty as potential mediators of change in comorbid depression in transdiagnostic CBT for anxiety. Journal of affective disorders. doi:10.1016/j.jad.2018.04.104

Thompson-Brenner, H., Boswell, J. F., Espel-Huynh, H., Brooks, G., \& Lowe, M. R. (2018). Implementation of transdiagnostic treatment for emotional disorders in residential eating disorder programs: A preliminary pre-post evaluation. Psychotherapy Research, 29(8), 1-17. doi:10.1080/10503307.2018.1446563

Trosper, S. E., Buzzella, B. A., Bennett, S. M., \& Ehrenreich, J. T. (2009). Emotion regulation in youth with emotional disorders: implications for a unified treatment approach. Clin Child Fam Psychol Rev, 12(3), 234-254. doi:10.1007/s10567-0090043-6

van der Heiden, C., Muris, P., \& van der Molen, H. T. (2012). Randomized controlled trial on the effectiveness of metacognitive therapy and intolerance-of-uncertainty therapy for generalized anxiety disorder. Behaviour research and therapy, 50(2), 100-109. doi:10.1016/j.brat.2011.12.005

Zemestani, M., Imani, M., \& Ottaviani, C. (2017). A Preliminary Investigation on the Effectiveness of Unified and Transdiagnostic Cognitive Behavior Therapy for Patients With Comorbid Depression and Anxiety. International Journal of Cognitive Therapy, 10(2), 175-185. doi:10.1521/ijct.2017. 10.2.175 